\title{
Pricing Vulnerable Option under Jump-Diffusion Model with Incomplete Information
}

\author{
Yang Jiahui, Zhou Shengwu (D), Zhou Haitao, and Guo Kaiqiang \\ School of Mathematics, China University of Mining and Technology, Xuzhou 221116, China \\ Correspondence should be addressed to Zhou Shengwu; zswcumt@163.com
}

Received 12 December 2018; Accepted 2 May 2019; Published 20 May 2019

Academic Editor: Giancarlo Consolo

Copyright (c) 2019 Yang Jiahui et al. This is an open access article distributed under the Creative Commons Attribution License, which permits unrestricted use, distribution, and reproduction in any medium, provided the original work is properly cited.

\begin{abstract}
In this paper, the closed-form pricing formula for the European vulnerable option with credit risk and jump risk under incomplete information was derived. Noise was introduced to the option writers assets while the underlying asset price and the value of corporation were assumed to follow the jump-diffusion processes. Finally the numerical experiment showed that jumps of underlying assets would increase the value of the option, but noise of corporation value was opposite.
\end{abstract}

\section{Introduction}

Credit risk is a kind of risk that is hard to analyze and manage quantitatively. Since there are no regulators like clearing firms to manage credit risk effectively in over-the-counter exchange, the greater probability of long position of option suffer from credit default risk in the OTC. Such options whose long positions exposed to both market risk and credit risk synchronously are called the vulnerable options.

The classical option pricing model has been presented by Black and Scholes [1], and the option pricing theory has made a breakthrough. Merton [2] constructed the corresponding option pricing model and gave the option pricing formula by assuming that the return of the underlying stock follows a continuous and jump mixed process. Johnson and Stulz [3] derived the pricing formula of the vulnerable option by assuming that the writers asset follows lognormal process and the underlying asset price follows lognormal diffusion process. Klein [4] defined an event of default occurring when the value of firm assets is less than a fixed boundary at maturity and assumed that asset of counterparty and options underlying asset are correlated, and the closed-form solution of the vulnerable options pricing was derived. Lando and Duffie [5] studied default intensity under imperfect information. Assuming that the asset value of the firm obeyed the geometric Brownian motion with noise, the conditional distribution of the asset value was obtained and default intensity can also be calculated. Goldberg and Giesecke [6] obtained the price of credit sensitive claims by establishing a credit model with assuming that the default threshold of the writer was a random variable independent of the firms value. Assuming the jump-diffusion process followed by asset prices which satisfied a partial integro-differential equation, Mayo [7] proposed a more efficient method for numerical evaluation of option prices. Lakner and Liang [8] analyzed the optimal investment strategy of credit default bonds based on structural model and reduced model by introducing the equivalent martingale measure. Based on model of Klein [4], under a Markov-modulated jump-diffusion model, Wang and Wang [9] considered the pricing of vulnerable European options. Xu et al. [10] have obtained the pricing model of vulnerable options under jump-diffusion assumptions about the underlying stock prices and firm values. Tian et al. [11] obtained the pricing model of vulnerable option and discussed the influence of jump on the value of vulnerable option by decomposing jumps in the underlying stock price and corporate value into two parts caused by the asset itself and by the market, respectively. Lyu and Zhang [12] derived vulnerable European option pricing formula with the timedependent for double jump-diffusion process by assuming that the underlying stock price and the ratio of the writers asset to their debt are governed by jump-diffusion processes. Based on the reduced model, Wang et al. [13] obtained the closed-form solution of European vulnerable option 
under fractional Brownian motion with assumption that stock prices follow jump-diffusion model. The approximate analytic pricing formula of vulnerable American put options under jump-diffusion assumptions on the underlying asset and the assets of the counterparty were derived by Wang et al. [14]. Xu and Jia [15] used Tikhonov regularization method and an iterative algorithm to correct the volatility in jump-diffusion process option pricing model. Liu et al. [16] introduced the jump-diffusion process into the compound option and obtained the corresponding pricing formula.

In this paper, we assume that investors can only obtain incomplete information at some specific time points in the structural model. Based on this, we investigate the pricing model of vulnerable option under imperfect information. A structured model for the pricing of vulnerable option is built and the closed-form solution is given in Section 2 under jump-diffusion model assumptions about stock prices and values of option short asset. In Section 3, the influence of pricing parameters on option value is studied by numerical experiments.

\section{Pricing Vulnerable Option}

In real financial markets, the jumps in financial asset prices are triggered by policy and big news. In this section, we investigated the pricing of European vulnerable option with jump-diffusion model under incomplete information.

Assume that $\mathscr{T}:=[0, T]$ is the limited time level, and $\left(\Omega, \mathscr{F}, \mathscr{F}_{t \in \mathscr{T}}, Q\right)$ is the complete probability space where $Q$ is the risk neutral probability measure. The underlying stock price $S_{t}$ and the value of option writers asset $V_{t}$ are assumed to be governed by the following stochastic differential equations, respectively:

$$
\begin{aligned}
& \frac{d S_{t}}{S_{t}}=\left(r-k_{1} \lambda_{1}\right) d t+\sigma_{1} d W_{1 t}+\left(e^{Z_{1 t}}-1\right) d N_{1 t} \\
& \frac{d V_{t}}{V_{t}}=\left(r-k_{2} \lambda_{2}\right) d t+\sigma_{2} d W_{2 t}+\left(e^{Z_{2 t}}-1\right) d N_{2 t}
\end{aligned}
$$

where $r$ is the risk-free interest rate, $\sigma_{1}$ is the volatility of stock prices, $\sigma_{2}$ is the volatility of option writers asset values, $W_{1 t}$ and $W_{2 t}$ are standard Brownian motions with a correlation coefficient $\rho_{12}, N_{1 t}$ and $N_{2 t}$ are Poisson processes with intensities of $\lambda_{1}$ and $\lambda_{2}$ respectively, $Z_{i t} \sim N\left(\mu_{i}, \sigma_{i J}^{2}\right)$, $k_{i}=e^{\mu_{i}+(1 / 2) \sigma_{i J}^{2}}, i=1,2$. We suppose that $\left(W_{1 t}, W_{2 t}\right), N_{1 t}, N_{2 t}$, $Z_{1 t}, Z_{2 t}$ are independent.

It is assumed that the long option of the position cannot obtain all the state information about the assets of the short position in time and only receive incomplete information at some specific moments $t_{1}, t_{2}, \cdots\left(t_{i}<t_{i+1}, i=1,2, \cdots\right)$. The asset value of the short position $\widehat{V}_{t}$ is also assumed with noise existing at each observation moment $t$. We extend the assumption for noise of assets by Lando and Luffie [4] (2001):

$$
\ln \widehat{V}_{t}=\ln V_{t}+U(t)=\ln V_{t}-\frac{1}{2} \sigma_{3}^{2} T+\int_{0}^{T} \sigma_{3} d W_{3 u},
$$

where noise $U(t) \sim N\left(-(1 / 2) \sigma_{3}^{2} T, \sigma_{3}^{2}\right), \sigma_{3}$ is a positive constant, $W_{3 t}$ is a standard Brownian motion, and we suppose that $\left(W_{1 t}, W_{2 t}\right), W_{3 t}, N_{1 t}, N_{2 t}, Z_{1 t}$, and $Z_{2 t}$ are independent.

The default happens when the asset value of the short position reaches the default boundary. When the default occurs, the recovery rate of the short assets is $(1-w) \widehat{V}_{T} / D$, where $D$ is the default threshold, and $w$ presents the percentage of cost resulting from short position bankruptcy to value of option writer. Under the risk neutral probability measure, the value of European vulnerable option at time zero is driven by the following equation:

$$
\begin{aligned}
& C(0, T)=E\left[e^{-r T}\left(S_{T}-K\right)^{+}\right. \\
& \left.\quad\left(1_{\left\{\widehat{V}_{T} \geq D^{*}\right\}}+\frac{(1-w) \widehat{V}_{T}}{D} 1_{\left\{\widehat{V}_{T}<D^{*}\right\}}\right) \mid \mathscr{F}_{0}\right]
\end{aligned}
$$

where $D^{*}$ is the default boundary which is equal to the sum of the default threshold $D$ and other debts of short option position.

Theorem 1. Assume that the underlying stock price $S_{t}$ and the value of option writers assets $V_{t}$ are driven by jump-diffusion models (1) and (2), respectively. The long position of option receives some information of the option writer at particular time points. The reporting value of the option writer $V_{t}$ is given by (3). Then the value of the European vulnerable option with strike price $K$ and maturity $T$ is

$$
C(0, T)=\sum_{n=0}^{\infty} \sum_{m=0}^{\infty} \frac{\left(\lambda_{1} T\right)^{n}\left(\lambda_{2} T\right)^{m} e^{-\lambda_{1} T-\lambda_{2} T}}{n ! m !} C_{n, m}
$$

where

$$
\begin{aligned}
C_{n, m}= & S_{0} e^{-k_{1} \lambda_{1} T+n \mu_{1}+(1 / 2) n \sigma_{1 J}^{2}} N\left(a_{1}(n), a_{2}(m), \bar{\rho}(n, m)\right)-K N\left(b_{1}(n), b_{2}(m), \bar{\rho}(n, m)\right) \\
& +\frac{1-w}{D} S_{0} \widehat{V}_{0} e^{r T-k_{1} \lambda_{1} T+n \mu_{1}+(1 / 2) n \sigma_{1 J}^{2}-k_{2} \lambda_{2} T+m \mu_{2}+(1 / 2) m \sigma_{2 J}^{2}-\bar{\rho} \sqrt{\sigma_{1}^{2} T+n \sigma_{1 J}^{2}} \sqrt{\sigma_{V}^{2} T+m \sigma_{2 J}^{2}}} \\
& \times N\left(c_{1}(n, m), c_{2}(n, m),-\bar{\rho}(n, m)\right)-\frac{1-w}{D} K \widehat{V}_{0} e^{-k_{2} \lambda_{2} T+m \mu_{2}+(1 / 2) m \sigma_{2 J}^{2}} N\left(d_{1}(n, m), d_{2}(m),-\bar{\rho}(n, m)\right), \\
b_{1}(n)= & \frac{\ln \left(S_{0} / K\right)+\left(r-(1 / 2) \sigma_{1}^{2}-k_{1} \lambda_{1}\right) T+n \mu_{1}}{\sqrt{n \sigma_{1 J}^{2}+\sigma_{1}^{2} T}},
\end{aligned}
$$




$$
\begin{aligned}
b_{2}(m) & =\frac{\ln \left(V_{0} / D^{*}\right)+\left(r-(1 / 2) \sigma_{2}^{2}-(1 / 2) \sigma_{3}^{2}-k_{2} \lambda_{2}\right) T+m \mu_{2}}{\sqrt{m \sigma_{2 J}^{2}+\sigma_{V}^{2} T}}, \\
a_{1}(n) & =b_{1}(n)+\sqrt{\sigma_{1}^{2} T+n \sigma_{1 J}^{2}}, \\
a_{2}(n, m) & =b_{2}(m)+\bar{\rho} \sqrt{\sigma_{1}^{2} T+n \sigma_{1 J}^{2}}, \\
c_{1}(n, m) & =b_{1}(n)+\sqrt{\sigma_{1}^{2} T+n \sigma_{1 J}^{2}}-\bar{\rho} \sqrt{\sigma_{V}^{2} T+m \sigma_{2 J}^{2}} \\
c_{2}(n, m) & =-b_{2}(n)+\bar{\rho} \sqrt{\sigma_{1}^{2} T+n \sigma_{1 J}^{2}}-\sqrt{\sigma_{V}^{2} T+m \sigma_{2 J}^{2}} \\
d_{1}(n, m) & =b_{1}(n)-\bar{\rho} \sqrt{\sigma_{V}^{2} T+m \sigma_{2 J}^{2}}, \\
d_{2}(m) & =-b_{2}(m)-\sqrt{\sigma_{V}^{2} T+m \sigma_{2 J}^{2}}, \\
\sigma_{V}^{2} & =\sigma_{2}^{2}+\sigma_{3}^{2}, \\
\bar{\rho} & =\frac{\rho_{12} \sigma_{1} \sigma_{2} T}{\sqrt{n \sigma_{1 J}^{2}+\sigma_{1}^{2} T} \sqrt{m \sigma_{2 J}^{2}+\sigma_{V}^{2} T}} \\
N(x, y, \rho) & \int_{-\infty}^{x} \int_{-\infty}^{y} \frac{1}{2 \pi \sqrt{1-\rho^{2}}} e^{(-1 / 2 \rho)\left(x^{2}-2 \rho x y+y^{2}\right)} d x d y .
\end{aligned}
$$

Proof. Consider (1), (2), and (3); by use of Itô's formula, we have

$$
\begin{aligned}
\ln S_{T}= & \ln S_{0}+\left(r-\frac{1}{2} \sigma_{1}^{2}-k_{1} \lambda_{1}\right) T+\int_{0}^{T} \sigma_{1} d W_{1 u} \\
& +\sum_{i=1}^{N_{1 T}} Z_{1 T}^{(i)}, \\
\ln V_{T}= & \ln V_{0}+\left(r-\frac{1}{2} \sigma_{2}^{2}-k_{2} \lambda_{2}\right) T+\int_{0}^{T} \sigma_{2} d W_{2 u} \\
& +\sum_{i=1}^{N_{2 T}} Z_{2 T}^{(i)}, \\
\ln \widehat{V}_{T}= & \ln \widehat{V}_{0}+\left(r-\frac{1}{2} \sigma_{2}^{2}-\frac{1}{2} \sigma_{3}^{2}-k_{2} \lambda_{2}\right) T \\
& +\int_{0}^{T} \sigma_{2} d W_{2 u}+\int_{0}^{T} \sigma_{3} d W_{3 \mathrm{u}}+\sum_{i=1}^{N_{2 T}} Z_{2 T}^{(i)},
\end{aligned}
$$

and under the condition $\mathscr{G}_{T}^{(n, m)}:=N_{T}^{1}=n, N_{T}^{2}=m$, the jumping times of $S_{T}$ and $V_{T}$ are $n$ and $m$, respectively, and the jumping time of $\widehat{V}_{T}$ is $m$. So

$$
+\sum_{i=1}^{n} Z_{1 T}^{(i)}
$$

$$
\begin{aligned}
\ln V_{T, m}= & \ln V_{0}+\left(r-\frac{1}{2} \sigma_{2}^{2}-k_{2} \lambda_{2}\right) T+\int_{0}^{T} \sigma_{2} d W_{2 u} \\
& +\sum_{i=1}^{m} Z_{2 T}^{(i)}
\end{aligned}
$$

$$
\begin{aligned}
\ln \widehat{V}_{T, m}= & \ln \widehat{V}_{0}+\left(r-\frac{1}{2} \sigma_{2}^{2}-\frac{1}{2} \sigma_{3}^{2}-k_{2} \lambda_{2}\right) T \\
& +\int_{0}^{T} \sigma_{2} d W_{2 u}+\int_{0}^{T} \sigma_{3} d W_{3 u}+\sum_{i=1}^{m} Z_{2 T}^{(i)}
\end{aligned}
$$

According to that, $\ln \left(S_{T, n} / S_{0}\right), \ln \left(V_{T, m} / V_{0}\right), \ln \left(\widehat{V}_{T, m} / \widehat{V}_{0}\right)$ are following normal distribution. In sample space $\Omega=$ $\bigcup_{n=0}^{\infty} \bigcup_{m=0}^{\infty} \mathscr{G}_{T}^{(n, m)}$, applying the total expectation formula, $C(0, T)$ can be rewritten as

$$
\ln S_{T, n}=\ln S_{0}+\left(r-\frac{1}{2} \sigma_{1}^{2}-k_{1} \lambda_{1}\right) T+\int_{0}^{T} \sigma_{1} d W_{1 u} \quad C(0, T)=e^{-r T} E\left[( S _ { T , n } - K ) ^ { + } \left(1_{\left\{\widehat{V}_{T, m} \geq D^{*}\right\}}\right.\right.
$$




$$
\begin{aligned}
& \left.\left.+\frac{(1-w) \widehat{V}_{T, m}}{D} 1_{\left\{\widehat{V}_{T, m}<D^{*}\right\}}\right) 1_{\{\omega \in \Omega\}}\right]=\sum_{n=0}^{\infty} \sum_{m=0}^{\infty} Q\left(N_{T}^{1}\right. \\
& \left.=n, N_{T}^{2}=m\right) e^{-r T} E\left[\left(S_{T, n}-K\right)^{+}\right. \\
& \left.\cdot\left(1_{\left\{\widehat{V}_{T, m} \geq D^{*}\right\}}+\frac{(1-w) \widehat{V}_{T, m}}{D} 1_{\left\{\widehat{V}_{T, m}<D^{*}\right\}}\right)\right] \\
& =\sum_{n=0}^{\infty} \sum_{m=0}^{\infty} \frac{\left(\lambda_{1} T\right)^{n}\left(\lambda_{2} T\right)^{m} e^{-\lambda_{1} T-\lambda_{2} T}}{n ! m !} C_{n, m},
\end{aligned}
$$

where

$$
C_{n, m}=e^{-r T} E\left[\left(S_{T, n}-K\right)^{+}\right.
$$

$$
\begin{aligned}
& \left.\cdot\left(1_{\left\{\widehat{V}_{T, m} \geq D^{*}\right\}}+\frac{(1-w) \widehat{V}_{T, m}}{D} 1_{\left\{\widehat{V}_{T, m}<D^{*}\right\}}\right)\right] \\
& =e^{-r T}\left[A_{1}(n, m)+A_{2}(n, m)+A_{3}(n, m)\right. \\
& \left.+A_{4}(n, m)\right]
\end{aligned}
$$

here

$$
\begin{aligned}
& A_{1}(n, m)=E\left[S_{T, n} 1_{\left\{S_{T, n} \geq K, \widehat{V}_{T, m} \geq D^{*}\right\}}\right], \\
& A_{3}(n, m)=\frac{1-w}{D} E\left[S_{T, n} \widehat{V}_{T, m} 1_{\left\{S_{T, n} \geq K, \widehat{V}_{T, m}<D^{*}\right\}}\right], \\
& A_{2}(n, m)=-K E\left[1_{\left\{S_{T, n} \geq K, \widehat{V}_{T, m} \geq D^{*}\right\}}\right], \\
& A_{4}(n, m)=-\frac{1-w}{D} E\left[\widehat{V}_{T, m} 1_{\left\{S_{T, n} \geq K, \widehat{V}_{T, m}<D^{*}\right\}}\right],
\end{aligned}
$$

and in the Appendix, we have

$$
\begin{aligned}
& A_{1}(n, m)=S_{0} e^{\left(r-k_{1} \lambda_{1}\right) T+n \mu_{1}+(1 / 2) n \sigma_{1 J}^{2}} N\left(a_{1}(n), a_{2}(n, m) \bar{\rho}(n, m)\right), \\
& A_{2}(n, m)=-K N\left(b_{1}(n), b_{2}(m), \bar{\rho}(n, m)\right), \\
& A_{3}(n, m)=-\frac{1-w}{D} S_{0} \widehat{V}_{0} e^{\left(r-k_{1} \lambda_{1}\right) T+n \mu_{1}+(1 / 2) n \sigma_{1 J}^{2}+\left(r-k_{2} \lambda_{2}\right) T+m \mu_{2}+(1 / 2) m \sigma_{2 J}^{2}-\bar{\rho} \sqrt{\sigma_{1}^{2} T+n \sigma_{1 J}^{2}} \sqrt{\sigma_{V}^{2} T+M \sigma_{2 J}^{2}}} \\
& \cdot N\left(c_{1}(n, m), c_{2}(n, m),-\bar{\rho}(n, m)\right), \\
& A_{4}(n, m)=-\frac{1-w}{D} K \widehat{V}_{0} e^{\left(r-k_{2} \lambda_{2}\right) T+m \mu_{2}+(1 / 2) m \sigma_{2 J}^{2}} N\left(d_{1}(n, m), d_{2}(m),-\bar{\rho}(n, m)\right),
\end{aligned}
$$

take those to (13), the pricing formula (5) of European vulnerable call option at zero time under imperfect information.

Corollary 2 (B-S model). If $D^{*}=0, \lambda_{1}=\lambda_{2}=0, n=m=0$, then the pricing formula (5) can be simplified to $B-S$ pricing formula of European vulnerable call option:

$$
C(0, T)=S_{0} N\left(A_{1}\right)-K e^{-r T} N\left(B_{1}\right)
$$

where

$$
\begin{aligned}
& A_{1}=\frac{\left[\ln \left(S_{0} / K\right)+\left(r+(1 / 2) \sigma_{1}^{2}\right) T\right]}{\sigma_{1} T}, \\
& B_{1}=A_{1}-\sigma_{1} \sqrt{T} .
\end{aligned}
$$

Corollary 3 (Merton's model). If $D^{*}=0$, then the pricing formula (5) can be rewritten as pricing formula of European vulnerable call option under Merton jump-diffusion model:

$$
C(0, T)=\sum_{n=0}^{\infty} \frac{\left(\lambda_{1} T\right)^{n} e^{-\lambda_{1} T}}{n !}
$$

$$
\begin{aligned}
& \cdot\left[S_{0} e^{-k_{1} \lambda_{1} T+n \mu_{1}+(1 / 2) n \sigma_{1 J}^{2}} N\left(a_{1}(n)\right)\right. \\
& \left.-K e^{-r T} N\left(b_{1}(n)\right)\right] .
\end{aligned}
$$

Corollary 4 (Klein's model). If $\lambda_{1}=0, n=0, \lambda_{2}=0, m=$ $0, \sigma_{3}=0$, then the pricing formula (5) can be reduced to pricing formula of European call option under model of Klein with complete information:

$$
\begin{aligned}
& C(0, T)=\left(S_{0} N\left(A_{1}, A_{2}, \rho_{12}\right)-K e^{-r T} N\left(B_{1}, B_{2}, \rho_{12}\right)\right. \\
& +\frac{1-w}{D} S_{0} V_{0} e^{r T+\rho_{12} \sigma_{1} \sigma_{2} T} N\left(C_{1}, C_{2},-\rho_{12}\right) \\
& \left.\quad-\frac{1-w}{D} K V_{0} N\left(D_{1}, D_{2},-\rho_{12}\right)\right)
\end{aligned}
$$

where

$$
\begin{aligned}
& B_{1}=\frac{\left[\ln \left(S_{0} / K\right)+\left(r-(1 / 2) \sigma_{1}^{2}\right) T\right]}{\sigma_{1} T}, \\
& B_{2}=\frac{\left[\ln \left(V_{0} / D^{*}\right)+\left(r-(1 / 2) \sigma_{2}^{2}\right) T\right]}{\sigma_{2} T},
\end{aligned}
$$




$$
\begin{aligned}
& A_{1}=B_{1}+\sigma_{1} \sqrt{T}, \\
& A_{2}=B_{2}+\rho_{12} \sigma_{1} \sqrt{T}, \\
& C_{1}=B_{1}+\left(\sigma_{1}+\rho_{12} \sigma_{2}\right) \sqrt{T}, \\
& C_{2}=-B_{2}-\left(\sigma_{2}+\rho_{12} \sigma_{1}\right) \sqrt{T}, \\
& D_{1}=B_{1}+\rho_{12} \sigma_{2} \sqrt{T}, \\
& D_{2}=-B_{2}-\sigma_{2} \sqrt{T} .
\end{aligned}
$$

\section{Numerical Experiment}

In this section, we take the quantitative experimenting approach to analyze the impact of various parameters in pricing formula (5) while taking the Black-Scholes model (1973), the Merton's jump-diffusion model (1976), and the Klein's vulnerable B-S model (1996) as basic objects for constructive research. The main purpose of these three models we chose is to observe the effect of noise introduced on option value and the influence of market price of jump risk is priced on option value. We suppose the parameters of formula (5) taking values are as follows:

$$
\begin{aligned}
S_{0} & =10, \\
\widehat{V}_{0} & =10, \\
K & =10, \\
D^{*} & =10, \\
D & =10, \\
T & =1, \\
r_{0} & =0.02, \\
\sigma_{1} & =0.3, \\
\sigma_{2} & =0.3, \\
\sigma_{3} & =0.3, \\
\lambda_{1} & =5 \\
\lambda_{2} & =5 \\
\mu_{1} & =0, \\
\mu_{2} & =0, \\
\sigma_{1 J} & =0.1, \\
\sigma_{2 J} & =0.1, \\
W & =0.4 .
\end{aligned}
$$

Firstly, we compare the option value under pricing model of vulnerable option under incomplete information with option value under traditional model such as B-S model, Merton's jump-diffusion model, and Klein's model. As shown

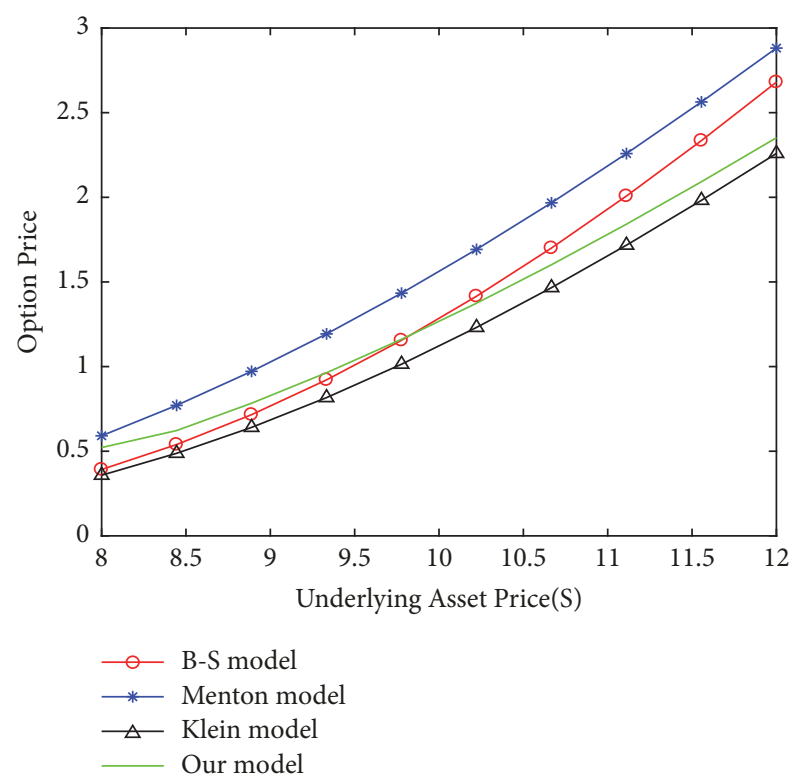

FIgURE 1: The price of vulnerable call option with incomplete information and other three classical models.

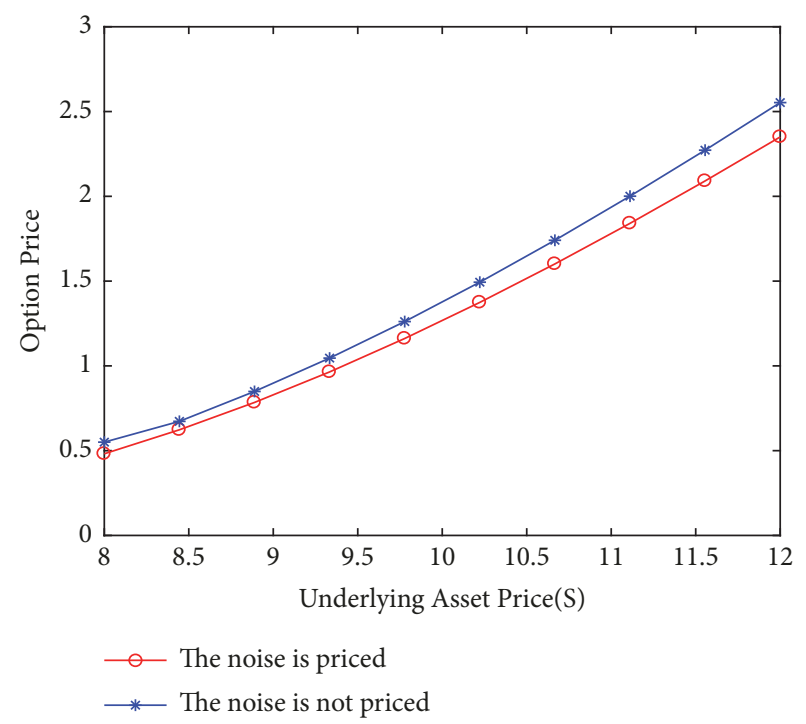

FIGURE 2: The influence of noise on vulnerable call option with incomplete information.

in Figure 1, the value of vulnerable option under incomplete information is lower than the option value under Merton's jump-diffusion model, but it is higher than the option value under Klein's model. There is a cross between the value of vulnerable option under imperfect information and the option value under classical B-S model which shows that the introduction of jump and noise factor makes the vulnerable option show higher value when the underlying stock price is lower.

Secondly, influence of introducing noise to the value of vulnerable options under incomplete information is investigated. From Figure 2, it is easy to find that, due to 


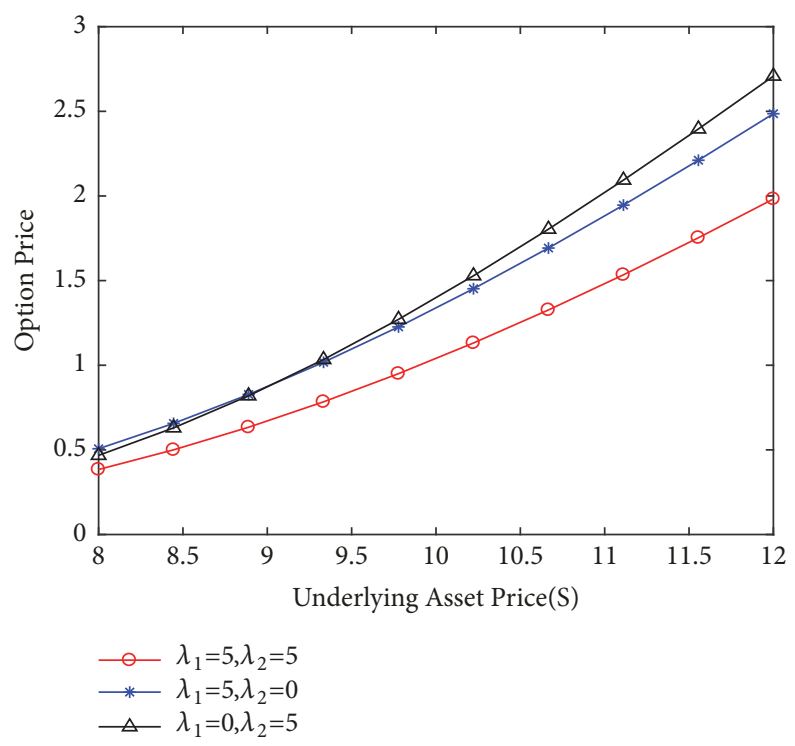

FIGURE 3: The influence of jump on vulnerable call option with incomplete information.

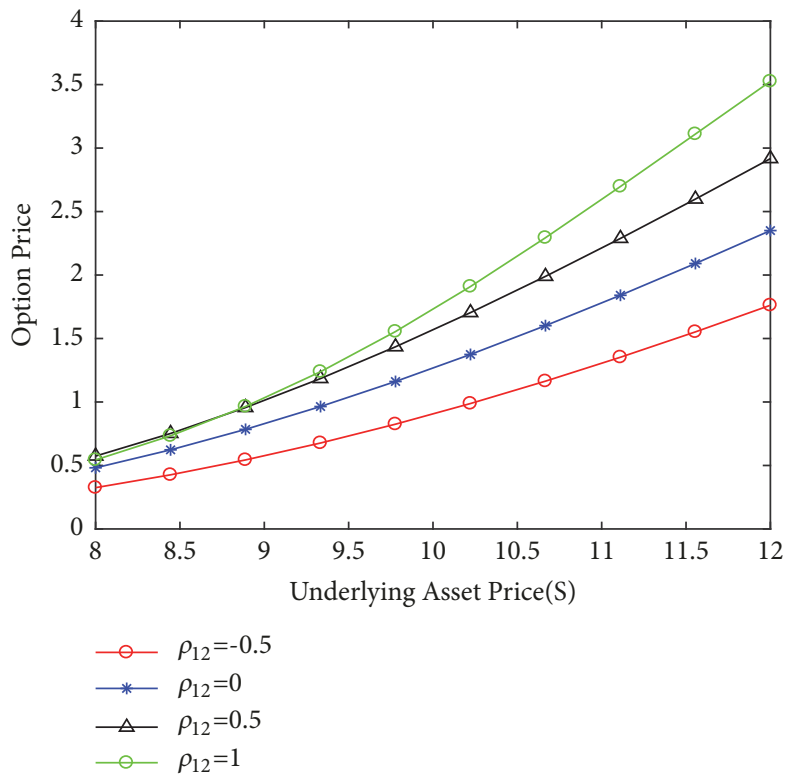

FIGURE 4: The influence of correlation coefficient $\rho_{12}$ on vulnerable call option with incomplete information.

the introduction of noise, the value of vulnerable options increases under incomplete information. It also shows that ignoring the factors that investors cannot always observe the changes of option writer assets may overestimate the value of option in the financial model.

The impact of jump in underlying stock and option writers assets on the value of vulnerable option under incomplete information is analyzed. When the jump intensities are $\lambda_{1}=$ $5, \lambda_{2}=0 ; \lambda_{1}=0, \lambda_{2}=5 ; \lambda_{1}=5, \lambda_{2}=5$, three different situations are shown in Figure 3. $\lambda_{1}=5, \lambda_{2}=0$ is used to explain jump changes caused by major events in the underlying stock. Analogously, $\lambda_{1}=0, \lambda_{2}=5$ is used to explain the sudden jump changes in option writer assets; $\lambda_{1}=$ $5, \lambda_{2}=5$ is used to explain the huge changes in underlying stock and option writer assets. It can be seen from Figure 3 that the jump of the underlying stock price increases the value of the vulnerable option under incomplete information, while the jump of the option writer assets value will reduce the value of the vulnerable option under incomplete information.

Finally, we study the influence of correlation coefficient between Brownian motions on option value of vulnerable option model under incomplete information. The effect of the correlation coefficient $\rho_{12}$ on option value is shown in Figure 4 . When the price of underlying stock is lower, the change of correlation coefficient has little influence on option value. With the increase of underlying stock price, the change of correlation coefficient has greater influence on option value. In addition, it can be seen that when the underlying 
stock price is given, the value of the option would rise with the increase of correlation coefficient. It also shows that the option value is positively correlated with the correlation coefficient.

\section{Conclusion}

In this paper, suppose that the underlying stock price and the value of the option writers asset are following jump-diffusion processes, and the closed-form solution of the European vulnerable option pricing with credit risk under incomplete information was obtained. We consider the condition of incomplete information, extend the assumption of Lando and Luffie [5] about asset noise, develop the framework of Klein, and obtain the pricing model of the European vulnerable option. In this model, we consider jump risk as unsystematic risk and compare the value of the European vulnerable option model under incomplete information to the value of the European vulnerable option under B-S model, Merton's jump-diffusion model, and Klein's model. In the numerical part, we derive that the jump of the underlying stock price will increase the option value, and the jump of the value of the option writer asset will reduce the value of the option.

\section{Appendix}

Suppose that $\xi_{1}$ and $\xi_{2}$ are standard normal distributions, and their correlation coefficient is $\bar{\rho}=$ $\rho_{12} \sigma_{1} \sigma_{2} T / \sqrt{n \sigma_{1 J}^{2}+\sigma_{1}^{2} T} \sqrt{M \sigma_{2 J}^{2}+\sigma_{V}^{2} T}$, where $\sigma_{V}^{2}=\sigma_{2}^{2}+\sigma_{3}^{2}$; we have

$$
\begin{aligned}
& \ln S_{T, n}=\ln S_{0}+\left(r-\frac{1}{2} \sigma_{1}^{2}-k_{1} \lambda_{1}\right) T+n \mu_{1}+\sqrt{\sigma_{1}^{2} T+n \sigma_{1 j}^{2}} \xi_{1} \\
& \ln \widehat{V}_{T, m}=\ln \widehat{V}_{0}+\left(r-\frac{1}{2} \sigma_{2}^{2}-\frac{1}{2} \sigma_{3}^{2}-k_{2} \lambda_{2}\right) T+m \mu_{2}+\sqrt{\sigma_{V}^{2} T+m \sigma_{2 J}^{2}} \xi_{2}, \\
& M_{S}(n)=E\left(\ln \frac{S_{T, n}}{S_{0}}\right)=\left(r-\frac{1}{2} \sigma_{1}^{2}-k_{1} \lambda_{1}\right) T+n \mu_{1} \\
& M_{V}(m)=E\left(\ln \frac{V_{T, m}}{V_{0}}\right)=\left(r-\frac{1}{2} \sigma_{2}^{2}-k_{2} \lambda_{2}\right) T+m \mu_{2} \\
& M_{\widehat{V}}(m)=E\left(\ln \frac{\widehat{V}_{T, m}}{\widehat{V}_{0}}\right)=\left(r-\frac{1}{2} \sigma_{2}^{2}-\frac{1}{2} \sigma_{3}^{2}-k_{2} \lambda_{2}\right) T+m \mu_{2}, \\
& \operatorname{cov}\left(\ln \frac{S_{T, n}}{S_{0}}, \ln \frac{V_{T, m}}{V_{0}}\right)=\rho_{12} \sigma_{1} \sigma_{2} T \\
& A_{1}(n, m)=E\left[S_{T, n} 1_{\left\{S_{T, n} \geq K, \widehat{V}_{T, m} \geq D^{*}\right\}}\right] \\
& =S_{0} e^{M_{S}(n)} E\left[e^{-\sqrt{\sigma_{1}^{2} T+n \sigma_{1 J}^{2}}\left(-\xi_{1}\right)} 1_{\left\{-\xi_{1} \leq-\left(\ln \left(K / S_{0}\right)-M_{S}(n)\right) / \sqrt{\sigma_{1}^{2} T+n \sigma_{1 J}^{2}},-\xi_{2} \leq-\left(\ln \left(D^{*} / \widehat{V}_{0}\right)-M_{\widehat{V}}(m)\right) / \sqrt{\sigma_{V}^{2} T+m \sigma_{2 J}^{2}}\right]}\right] \\
& =S_{0} e^{M_{S}(n)} E\left[e^{-\sqrt{\sigma_{1}^{2} T+n \sigma_{1 J}^{2}}\left(-\xi_{1}\right)} 1_{\left\{-\xi_{1} \leq b_{1}(n),-\xi_{2} \leq b_{2}(m)\right\}}\right] \\
& =S_{0} e^{M_{S}(n)} \int_{-\infty}^{b_{1}(n)} \int_{-\infty}^{b_{2}(m)} e^{-\sqrt{\sigma_{1}^{2} T+n \sigma_{1 J}^{2}} x} \frac{1}{2 \pi \sqrt{1-\bar{\rho}^{2}}} e^{\left(1 /-2 \sqrt{1-\bar{\rho}^{2}}\right)\left(x^{2}-2 \bar{\rho} x y+y^{2}\right)} d x d y \\
& =S_{0} e^{M_{S}(n)} \int_{-\infty}^{b_{1}(n)} \int_{-\infty}^{b_{2}(m)} e^{(1 / 2)\left(\sigma_{1}^{2} T+n \sigma_{1 J}^{2}\right)} \frac{1}{2 \pi \sqrt{1-\bar{\rho}^{2}}} \\
& \times e^{\left(1 /-2\left(1-\bar{\rho}^{2}\right)\right)\left[\left(x+\sqrt{\sigma_{1}^{2} T+n \sigma_{1 J}^{2}}\right)^{2}-2 \bar{\rho}\left(x+\sqrt{\sigma_{1}^{2} T+n \sigma_{1 J}^{2}}\right)\left(y+\sqrt{\sigma_{1}^{2} T+n \sigma_{1 J}^{2}}\right)+\left(y+\sqrt{\sigma_{1}^{2} T+n \sigma_{1 J}^{2}}\right)^{2}\right]} d x d y \\
& =S_{0} e^{M_{S}(n)} \int_{-\infty}^{a_{1}(n)} \int_{-\infty}^{a_{2}(n, m)} e^{(1 / 2)\left(\sigma_{1}^{2} T+n \sigma_{1 J}^{2}\right)} \frac{1}{2 \pi \sqrt{1-\bar{\rho}^{2}}} e^{\left(1 /-2\left(1-\bar{\rho}^{2}\right)\right)\left(x^{2}-2 \bar{\rho} x y+y^{2}\right)} d x d y \\
& =S_{0} e^{\left(r-k_{1} \lambda_{1}\right) T+n \mu_{1}+(1 / 2) n \sigma_{1 j}^{2}} N\left(a_{1}(n), a_{2}(n, m), \bar{\rho}(n, m)\right) .
\end{aligned}
$$


$A_{2}(n, m), A_{3}(n, m)$, and $A_{4}(n, m)$ can be obtained in a similar way.

\section{Data Availability}

The data used to support the findings of this study are available from the corresponding author upon request.

\section{Conflicts of Interest}

The authors declare that they have no conflicts of interest.

\section{Acknowledgments}

This work was supported by the National Natural Science Foundation of China (No. 61304088) and the Fundamental Research Funds for the Central Universities (No. 2013QNA37).

\section{References}

[1] F. Black and M. Scholes, "The pricing of options corporate liabilities," Journal of Political Economy, vol. 81, pp. 637-659, 1973.

[2] R. C. Merton, "Option pricing when underlying stock returns are discontinuous," Journal of Financial Economics, vol. 3, no. 1-2, pp. 125-144, 1976.

[3] H. Johnson and R. Stulz, "The pricing of options with default risk," The Journal of Finance, vol. 42, no. 2, pp. 267-280, 1987.

[4] P. Klein, "Pricing black-scholes options with correlated credit risk," Journal of Banking \& Finance, vol. 20, no. 7, pp. 1211-1229, 1996.

[5] D. Lando and D. Duffie, "Term structures of credit spreads with incomplete accounting information," Econometrica, vol. 69, no. 3, pp. 633-664, 2001.

[6] K. Giesecke and L. R. Goldberg, "Forecasting default in the face of uncertainty," Journal of Derivatives, vol. 12, no. 1, pp. 14-25, 2003.

[7] P. Carr and A. Mayo, "On the numerical evaluation of option prices in jump diffusion processes," European Journal of Finance, vol. 13, no. 4, pp. 353-372, 2007.

[8] P. Lakner and W. Liang, "Optimal investment in a defaultable bond," Mathematics \& Financial Economics, vol. 1, no. 3-4, pp. 283-310, 2008.

[9] W. Wang and W. Wang, "Pricing vulnerable options under a Markov-modulated regime switching model," Communications in Statistics-Theory and Methods, vol. 39, no. 19, pp. 3421-3433, 2010.

[10] W. Xu, W. Xu, H. Li, and W. Xiao, "A jump-diffusion approach to modelling vulnerable option pricing," Finance Research Letters, vol. 9, no. 1, pp. 48-56, 2012.

[11] L. Tian, G. Wang, X. Wang, and Y. Wang, "Pricing vulnerable options with correlated credit risk under jump-diffusion processes," Journal of Futures Markets, vol. 34, no. 10, pp. 957-979, 2013.

[12] L. J. Lyu and X. Y. Zhang, "Time-dependent vulnerable European option pricing for double jump-diffusion process," Journal of East China Normal University. Natural Science, no. 1, pp. 1320, 2014.
[13] C. Wang, S. W. Zhou, and J. Y. Yang, "The pricing of vulnerable options in a fractional brownian motion environment," Discrete Dynamics in Nature and Society, vol. 2015, Article ID 579213, 10 pages, 2015.

[14] G. Wang, X. Wang, and Z. Liu, "Pricing vulnerable American put options under jump-diffusion processes," Probability in the Engineering and Informational Sciences, vol. 31, no. 2, pp. 121138, 2017.

[15] Z. Xu and X. Jia, “The calibration of volatility for option pricing models with jump diffusion processes," Applicable Analysis: An International Journal, vol. 98, no. 4, pp. 810-827, 2019.

[16] Y. Liu, I. Jiang, and W. Hsu, "Compound option pricing under a double exponential Jump-diffusion model," The North American Journal of Economics and Finance, vol. 43, pp. 30-53, 2018. 


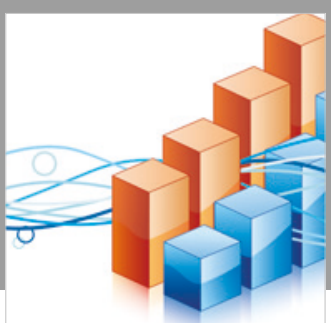

Advances in

Operations Research

\section{-n-m}
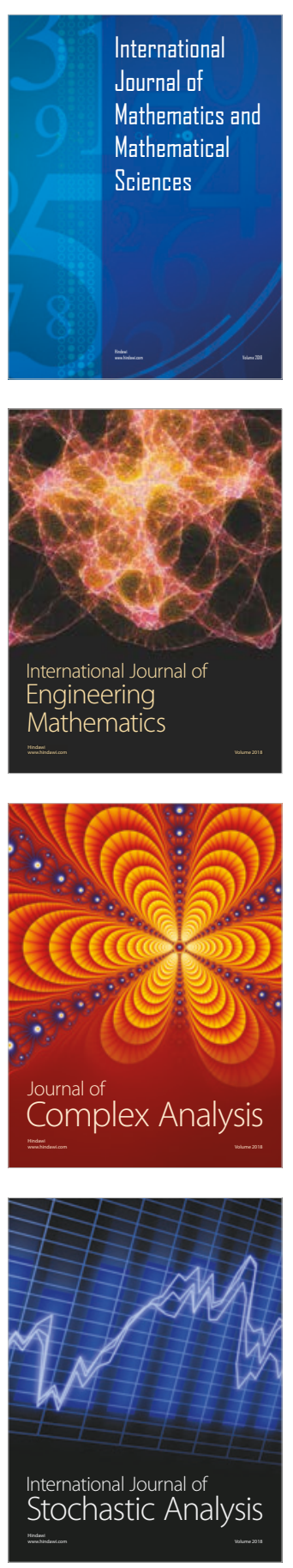
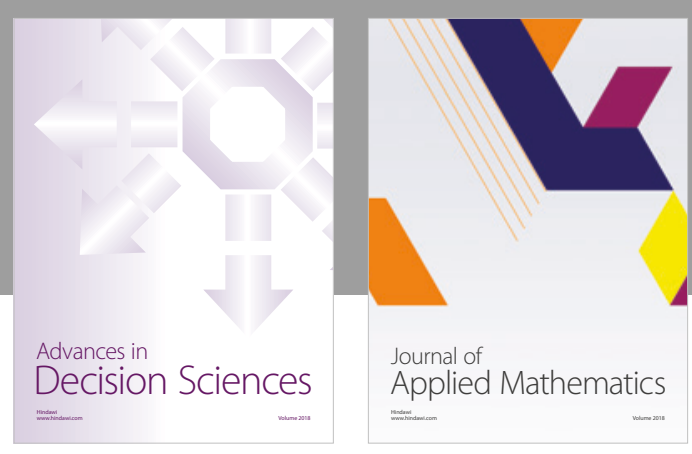

Journal of

Applied Mathematics
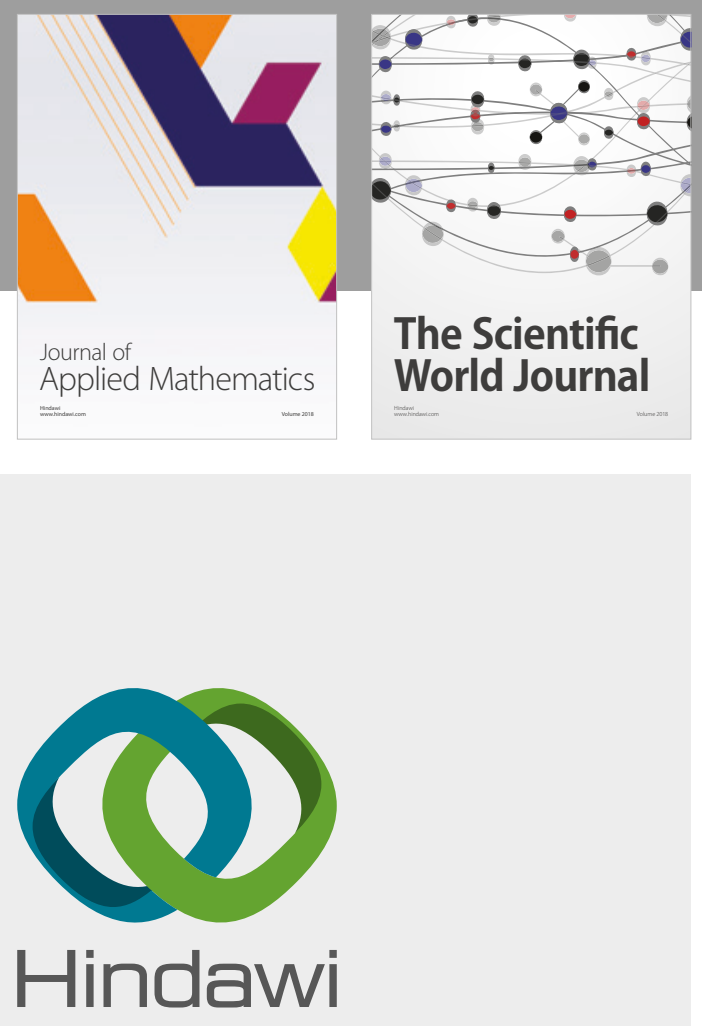

Submit your manuscripts at

www.hindawi.com

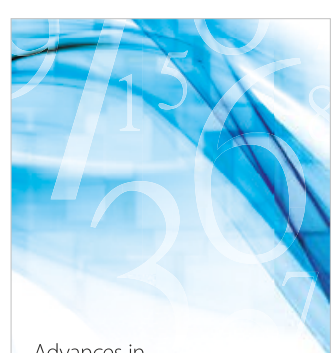

Advances in
Numerical Analysis
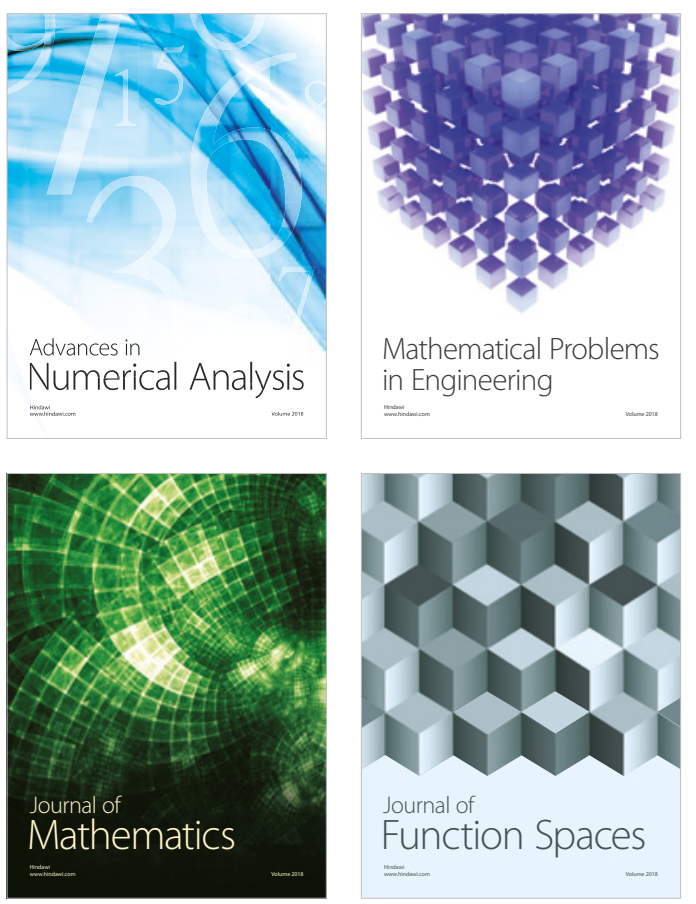

Mathematical Problems in Engineering

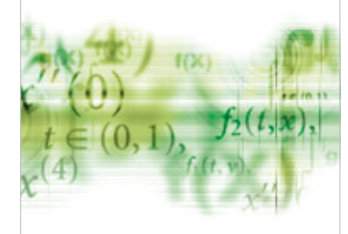

International Journal of

Differential Equations

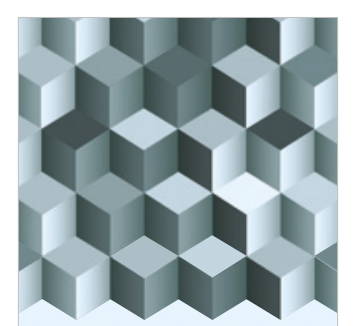

Journal of

Function Spaces

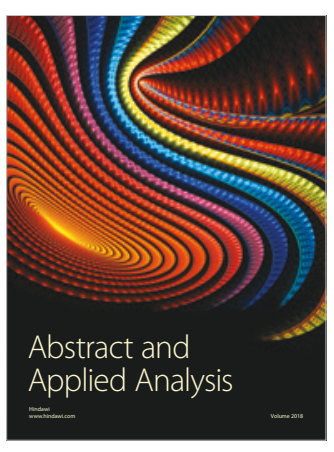

The Scientific

World Journal

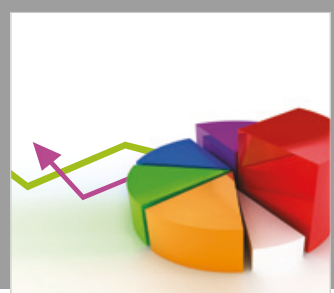

Journal of

Probability and Statistics
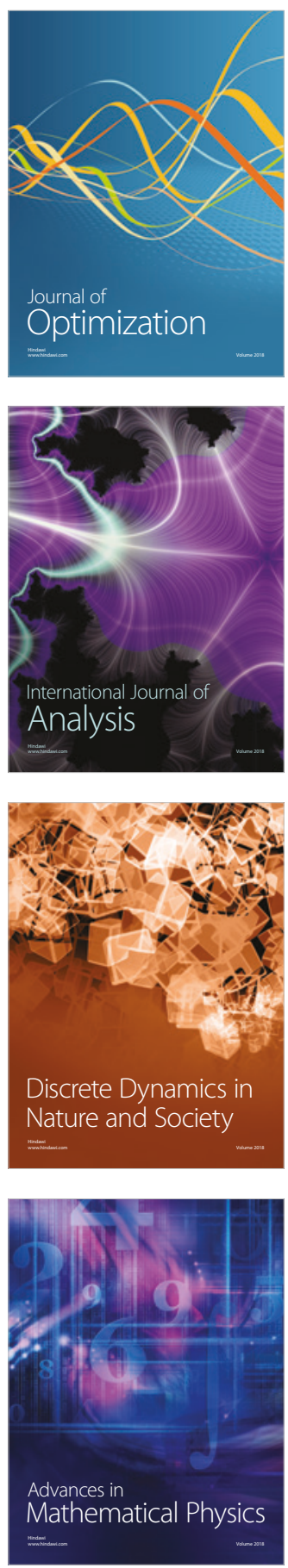